\title{
Desarrollo de competencias profesionales mediante la ejecución de proyectos académicos
}

\section{Developing professional skills through the execution of academic projects}

FLORES-CEDILLO, María Lisseth $\uparrow^{*}$, FLORES-CEDILLO, Ramiro Eduardo", MORALESBARBOSA, Ma. de la Luz y SIERRA-GUERRERO, Adela Marisol

Instituto Tecnológico Superior de San Luis Potosí, Capital, Departamento de Ingeniería Industrial, Carr. 57 México -Piedras Negras Km 189+100 No. 6501, C.P. 78421, San Luis Potosí, S.L.P.; México.

"Instituto de Profesionalización del Magisterio Potosino, Calle Ignacio Martínez No. 650, Col. Ladrillera, C.P. 78433, San Luis Potosí, S.L.P.; México.

ID $1^{\mathrm{er}}$ Autor: María Lisseth, Flores-Cedillo / ORC ID: 0000-0002-2693-6734, Researcher ID: E-6827-2018, CVU CONACYT ID: 426793

ID $1^{\text {er }}$ Coautor: Ramiro Eduardo, Flores-Cedillo / ORC ID: 0000-0002-08528956, Researcher ID: Y-1127-2019, CVU CONACYT ID: 775672

ID $2^{\text {do }}$ Coautor: Ma. de la Luz, Morales-Barbosa / ORC ID: 0000-0003-2536-5607, Researcher ID: S-6016-2018, CVU CONACYT ID: 890666

ID $3^{\text {er }}$ Coautor: Adela Marisol, Sierra-Guerrero / ORC ID: 0000-0003-3433-5012, Researcher ID: S-5688-2018, CVU CONACYT ID: 604622

DOI: $10.35429 / J E T \cdot 2019.7 .3 .13 .20$

Recibido: 28 de Enero, 2019; Aceptado 29 de Marzo, 2019

\section{Resumen}

En este trabajo, se evidencia la importancia de desarrollar proyectos académicos para el que los estudiantes desarrollen diversas competencias profesionales y potencien su capacidad aprendizaje para llevar la teoría a la práctica mediante la asesoría de un grupo de docentes con diferentes perfiles profesionales para que los proyectos dirigidos sean multidisciplinares. Se muestran ejemplos de proyectos desarrollados por diferentes instituciones educativas nacionales y extranjeras, de varios niveles educativos y programas de estudios; y se analizaron los tipos de competencias instrumentales, interpersonales $y$ sistémicas potenciadas por la ejecución proyectos académicos. Se estudió el impacto que tienen estos proyectos en el desarrollo y aplicación de competencias profesionales para promover la participación de estudiantes, docentes, directivos y autoridades educativas con este tipo de proyectos e incluso el poder realizar foros en los que se presenten los proyectos de diversas Instituciones para intercambiar ideas y enriquecer el conocimiento, además de fortalecer la vinculación. Finalmente, se presentan los hallazgos y conclusiones derivadas del estudio.

Competencias profesionales, Proyectos académicos, Instituciones educativas

\begin{abstract}
In this work, the importance of developing academic projects for which students develop various professional competencies and enhance their learning capacity to bring theory into practice through the advice of a group of teachers with different professional profiles so that the projects directed are evidenced that be multidisciplinary. Examples of projects developed by different national and foreign educational institutions, of various educational levels and curricula are shown; and the types of instrumental, interpersonal and systemic competencies enhanced by the execution of academic projects were analyzed. The impact of these projects on the development and application of professional competences to promote the participation of students, teachers, managers and educational authorities with this type of projects and even to be able to carry out forums in which the projects of various institutions were presented were studied. to exchange ideas and enrich knowledge, in addition to strengthening bonding. Finally, the findings and conclusions derived from the study are presented.
\end{abstract}

Professional competencies, Academic projects, Educational institutions

Citación: FLORES-CEDILLO, María Lisseth, FLORES-CEDILLO, Ramiro Eduardo, MORALES-BARBOSA, Ma. de la Luz y SIERRA-GUERRERO, Adela Marisol. Desarrollo de competencias profesionales mediante la ejecución de proyectos académicos. Revista Teoría Educativa. 2019 3-7: 13-20.

\footnotetext{
* Correspondencia del Autor (Correo electrónico: maria.flores@ tecsuperiorslp.edu.mx)

$\dagger$ Investigador contribuyendo como primer autor.
} 


\section{Introducción}

Aprendizaje por competencias requiere un modelo educativo bien estructurado que permita diseñar, implementar y evaluar el desarrollo de competencias a partir de distintas estrategias de enseñanza. Los avances sociales y tecnológicos actuales en el entorno educativo, deben replantearse cuestiones relacionadas con la labor docente, formando a los alumnos en capacidades y competencias que favorezcan que el discente se desenvuelva y actualice sus conocimientos de manera autónoma y ágil, adaptándose a los continuos avances en el saber, por lo que se les debe de dotar de herramientas necesarias que les permitan progresar y profundizar en su formación desde la construcción de un conocimiento adaptado a sus necesidades particulares (Navarro Soria, González Gómez, López Monsalve, \& Contreras Fontanillo, 2019).

Las Instituciones de Educación Superior (IES) deben vigilar el cumplimiento de los programas académicos, así como el nivel de avance de las diferentes competencias profesionales para poder fortalecer el perfil de sus egresados: Por lo cual el desarrollo de proyectos que les permitan poner en práctica los conocimientos adquiridos durante el tiempo de estancia en la Institución es una estrategia infalible para que puedan potenciar los tres saberes (saber, saber ser y saber hacer). Las competencias profesionales definen la capacidad productiva de un individuo que posee conocimientos, habilidades y actitudes que requiere para desempeñar un cierto tipo de trabajo (Clares \& Samanes, 2009), por lo que al impulsar su formación académica, es importante tomar en cuenta la empatía del estudiante con su entorno social. Asimismo, en otras IES se han experimentado cambios de paradigma educativo el cual ha otorgado una resignificación del binomio docencia-aprendizaje, pues el aprendizaje, antes basado en la adquisición de conocimientos, es ahora un elemento que contempla el saber, pero también el saber hacer, saber estar y saber ser. Este nuevo escenario ha supuesto retos, áreas de oportunidad, adaptación, capacitación e importantes modificaciones en las estrategias educativas desde la Educación Superior, con el fin de que las entidades universitarias formen profesionales activos, competentes y capaces de dar respuestas eficaces $\mathrm{y}$ eficientes a las demandas sociales (Ortuño, 2019).
Un factor importante en la enseñanza universitaria, está fuertemente vinculado a la gestión de la calidad, orientando e informando al alumnado para facilitar su integración a la vida universitaria y conseguir sus objetivos académicos (Galván, Pérez, Torres, \& Betancort, 2019).

En el caso de los Institutos Tecnológicos y su Modelo Educativo para el Siglo XXI, se orienta el proceso educativo central a la formación de profesionales que impulsen la actividad productiva en cada región del país, la investigación científica, la innovación tecnológica, la transferencia de tecnologías, la creatividad y el emprendedurismo para alcanzar un mayor desarrollo social, económico, cultural y humano (Modelo educativo para el siglo XXI: Formación y desarrollo de competencias profesionales, 2018).

Por lo anterior, los modelos educativos, hacen énfasis en el desarrollo de competencias y habilidades, permitiendo plantear estrategias diversas, entre ellas los proyectos académicos que permitan generar y afirmar conocimientos mediante el saber ser, saber hacer y saber convivir, vinculándolos con el entorno regional, nacional e internacional.

En este trabajo, se evaluó el impacto que tienen los proyectos académicos en la creación y potencialización de competencias profesionales en distintas IES bajo el asesoramiento de los docentes, avalados por las autoridades educativas y vinculados con instancias privadas, públicas y la sociedad en general.

El modelo de competencias profesionales establece tres niveles: las competencias básicas, las genéricas y las específicas o técnicas. En la Tabla 1, se muestra un listado general de cada estos niveles de competencias, los cuales permiten en los estudiantes adquirir nuevas habilidades, destrezas y conocimientos. 


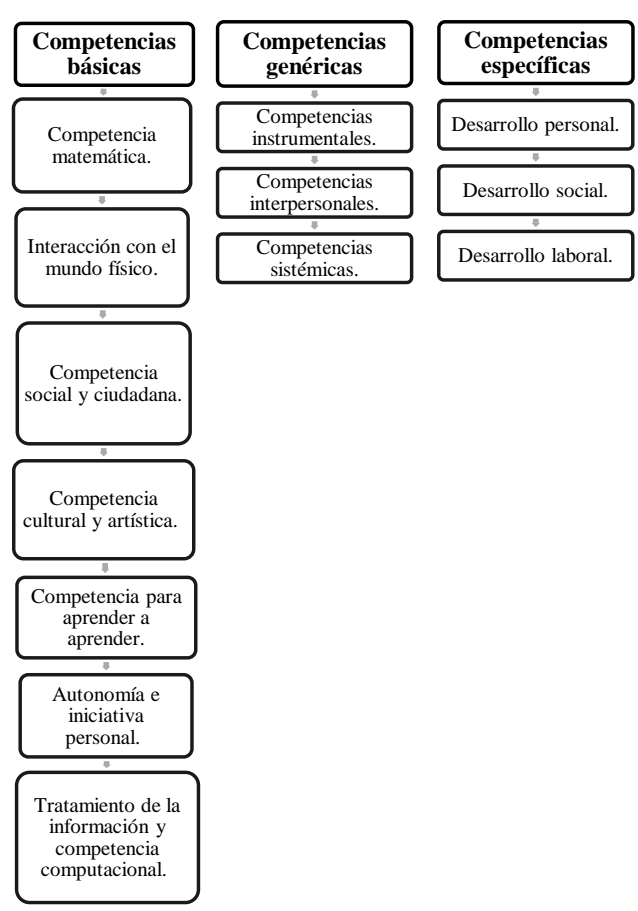

Tabla 1 Niveles básicos de competencias Fuente: elaboración propia

En la Tabla 2, se desglosan algunas competencias genéricas, las cuales, generalmente se mencionan desde los objetivos por lograr en cada una de las asignaturas de la malla curricular, así como en las instrumentaciones didácticas.

\begin{tabular}{|c|c|}
\hline Categoría & Competencias \\
\hline \multirow{8}{*}{ 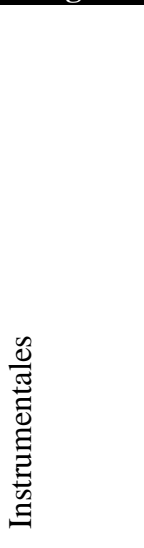 } & $\begin{array}{l}\text { Pensamiento reflexivo, lógico, analógico, } \\
\text { sistémico, crítico, creativo, práctico, } \\
\text { deliberativo, colegiado. }\end{array}$ \\
\hline & $\begin{array}{l}\text { Capacidad para el razonamiento crítico, lógico } \\
\text { matemático. }\end{array}$ \\
\hline & $\begin{array}{l}\text { Capacidad para resolver problemas dentro de } \\
\text { su área de estudio. }\end{array}$ \\
\hline & Capacidad de abstracción. \\
\hline & $\begin{array}{l}\text { Capacidad de crear y utilizar modelos que } \\
\text { reflejen situaciones reales. }\end{array}$ \\
\hline & $\begin{array}{l}\text { Capacidad de diseñar y realizar experimentos } \\
\text { y analizar e interpretar sus resultados. }\end{array}$ \\
\hline & Capacidad de análisis, síntesis y evaluación. \\
\hline & Aplicación de conocimientos en la práctica. \\
\hline \multirow{16}{*}{ 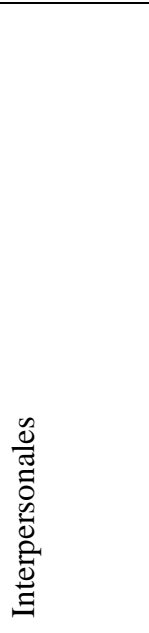 } & Toma de decisiones. \\
\hline & Organización del tiempo. \\
\hline & Comunicación verbal, no verbal y escrita. \\
\hline & Comunicación en un segundo idioma. \\
\hline & Automotivación y adaptación al entorno. \\
\hline & Sentido ético. \\
\hline & Análisis de problemas. \\
\hline & Análisis numérico. \\
\hline & Asunción de riesgos. \\
\hline & Iniciativa. \\
\hline & Nivel de detalle. \\
\hline & Planificación y organización. \\
\hline & Aprendizaje autónomo. \\
\hline & Tolerancia al estrés. \\
\hline & Tenacidad. \\
\hline & Compromiso. \\
\hline
\end{tabular}

\begin{tabular}{|c|c|}
\hline \multirow{13}{*}{ 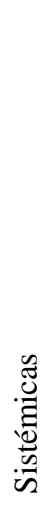 } & Creatividad. \\
\hline & Espíritu emprendedor. \\
\hline & Capacidad de crear e innovar. \\
\hline & Gestión por objetivos. \\
\hline & Gestión de proyectos. \\
\hline & Desarrollo de la calidad. \\
\hline & Estimulación intelectual. \\
\hline & Delega responsabilidades. \\
\hline & Búsqueda del logro. \\
\hline & Sensibilidad organizacional e interpersonal. \\
\hline & Capacidad de escuchar. \\
\hline & Flexibilidad. \\
\hline & Empatía. \\
\hline
\end{tabular}

Tabla 2 Ejemplos de competencias genéricas Fuente: elaboración propia

El reto del capital humano en México, se enmarca en los siguientes puntos: (1) se necesitan más profesionistas, (2) se necesitan profesionistas mejor preparados, y (3) se necesita una mayor coordinación entre lo que las IES enseñan y lo que las instituciones y empresas necesitan (Modelo educativo para el siglo XXI: Formación y desarrollo de competencias profesionales, 2018).

\section{Metodología}

Tomando en cuenta las razones que justifican la educación basada en competencias (EBC), se muestran distintos casos de estudio de proyectos académicos en IES y se realizó una evaluación de las diferentes competencias profesionales desarrolladas.

\section{Proyectos académicos}

Nombre de la IES: Instituto Tecnológico Superior de San Luis Potosí, Capital.

Programa Académico: Ingeniería Industrial.

Objetivo general:

Defender los proyectos académicos desarrollados durante el semestre enero-junio frente a jurados externos que puedan retroalimentar sus trabajos.

\section{Justificación:}

Anualmente se organiza un evento llamado "Programa de Experiencia Profesional" (PEP) al final del semestre, en el cual participan los estudiantes de las 4 ingenierías de la Institución asesorados y acompañados de docentes de cada área académica. 
Se presentan proyectos, los cuales son evaluados mediante jurados externos y se les imparten talleres, foros y conferencias para que puedan plantear sus inquietudes con gente que comparte sus experiencias profesionales (ver Figura 1).

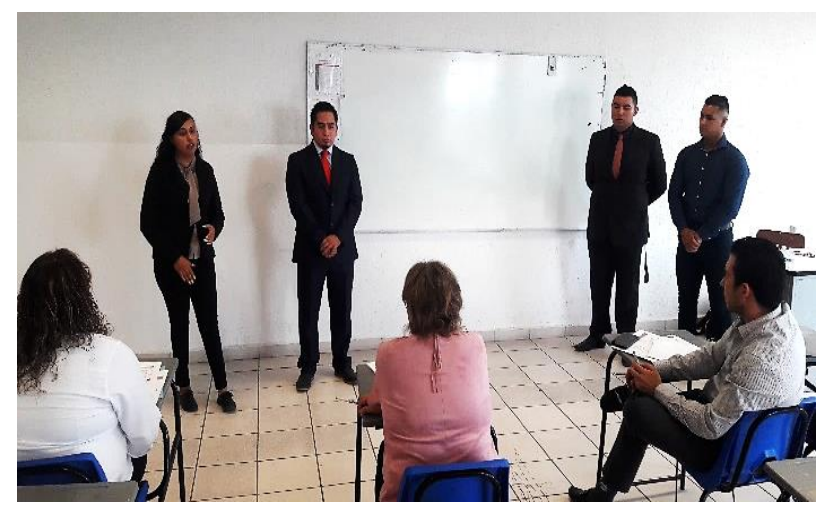

Figura 1 Defensa de proyectos en el evento PEP Fuente: («Tec Superior S.L.P.», 2019)

\section{Nombre de la IES}

Universidad Tecnológica de la costa Chica de Guerrero.

\section{Programas Académicos}

Ingeniería en procesos alimentarios, en mantenimiento industrial, en energías renovables, en Tecnologías de la información, e gestión y turismo, en gestión de capital humano, en gastronomía, en ingeniería en logística internacional.

\section{Objetivo general:}

Realizar proyectos integradores innovadores que permitan ofertar diferentes productos y servicios.

\section{Justificación:}

Se realizan estudios de factibilidad para el desarrollo y comercialización de productos y servicios (ver Figura 2).

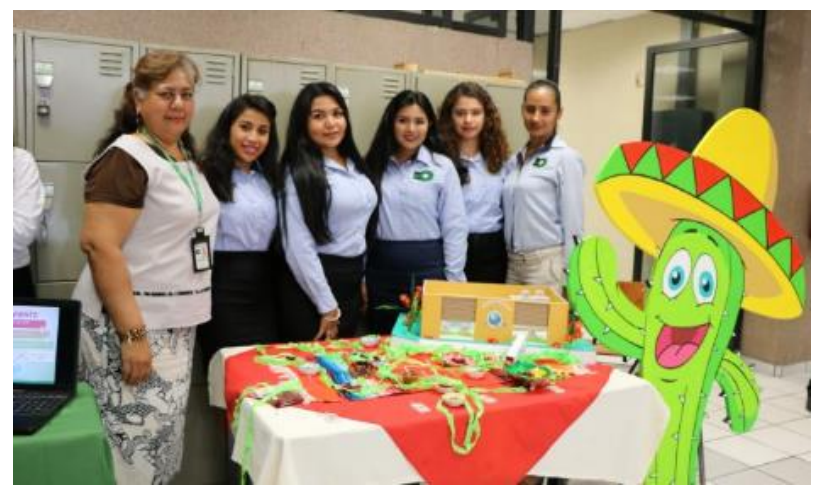

Figura 2 Presentación de proyectos integradores Fuente: («Universidad Tecnológica de la Costa Grande de Guerrero», 2017)

\section{Nombre de la IES}

Universidad San Francisco de Quito

\section{Programa Académico}

Maestría en Dirección de Empresas Constructoras e Inmobiliarias.

\section{Objetivo general}

Realizar un plan de negocios para un proyecto inmobiliario.

\section{Justificación}

A través de este trabajo se analizará la vialidad de la primera etapa del proyecto, que consiste en la construcción de 314 viviendas, y que ya están debidamente financiadas y en su etapa de ejecución. Este proyecto pretende colaborar con la empresa pública Emuvivienda para recomendar estrategias gerenciales, financieras, de marketing con el fin de obtener mejores resultados en ventas y que el proyecto termine sus 7 etapas según lo establecido en el proyecto de la empresa (ver Figura 3).

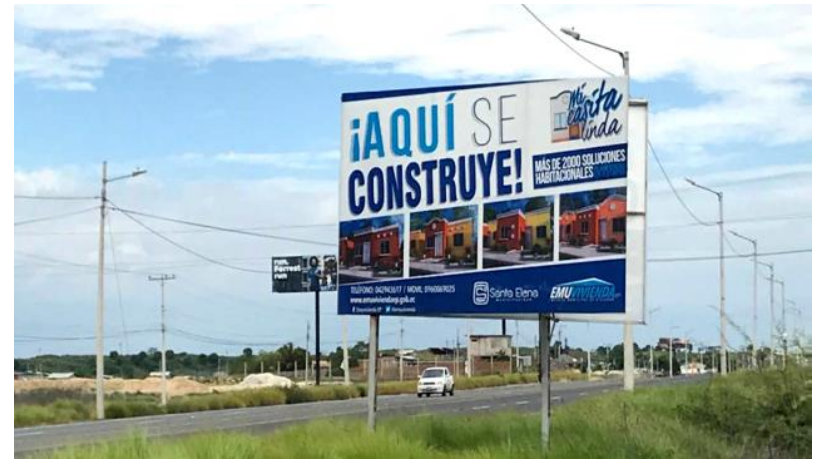

Figura 3 Ubicación de ingreso al proyecto Fuente: (Jarrín \& María, 2013) 


\section{IES de San Luis Potosí}

$6^{\circ}$ Encuentro de jóvenes investigadores.

Programas Académicos: Diversos.

Objetivo general:

Incentivar en los jóvenes el gusto por la ciencia de los jóvenes de las IES en el Estado de San Luis Potosí a través de la presentación de sus proyectos de investigación.

\section{Justificación:}

Se establecen categorías de participación en: Ciencias naturales e ingeniería; Ciencias sociales, educación y humanidades; Salud y ciencias agropecuarias, en las modalidades de prototipos, exposición de carteles y oral para que realicen la defensa de sus proyectos y compartan sus experiencias con otros jóvenes investigadores (ver Figura 4).

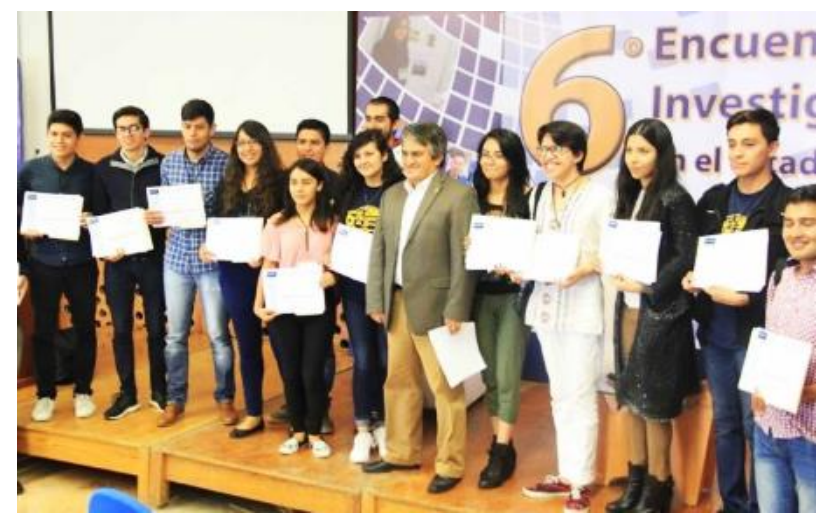

Figura 4 Presentación de proyectos de IES potosinas Fuente: («6o Encuentro de Jóvenes Investigadores en el estado de San Luis Potosí», 2018)

\section{Nombre de la IES: Facultad de ciencias económicas}

Programa Académicos: Ingeniería Empresarial.

Objetivo general:

Elaborar un análisis y formular estrategias para la creación de un producto o servicio innovador.

\section{Justificación:}

Este proyecto académico brinda las herramientas necesarias para entender los problemas de una organización y resolverlos por medio de la implementación de estrategias teóricas y tecnológicas, que le garanticen el crecimiento en un mercado competitivo.

Se estudia el modelo business canvas, integración de web y redes sociales; además se realiza análisis de resultados, entrevistas y prototipos (ver Figura 5).

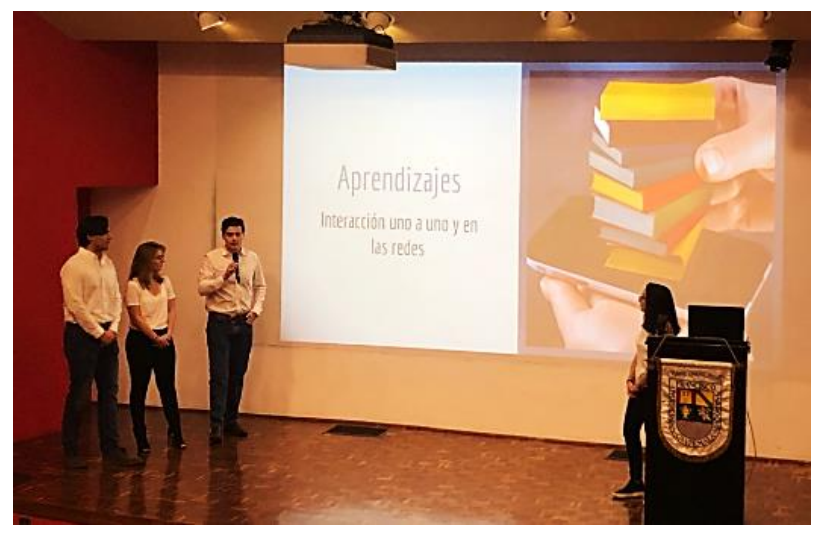

Figura 5 Presentación de proyectos de Ingeniería Empresarial

Fuente: («Estudiantes de Ingeniería presentaron proyectos de negocios - El Amigo de la Marro», 2019)

\section{Nombre de la IES: Universidad Mesoamericana}

Programa Académico: Licenciatura en Administración educativa

Objetivo general:

Presentar proyectos educativos como parte del curso de planteamiento educativo.

Justificación:

Realizar y presentar diversos proyectos educativos planteando su misión, visión y valores e imagen para validad las competencias de las diferentes asignaturas del programa educativo cursado. 


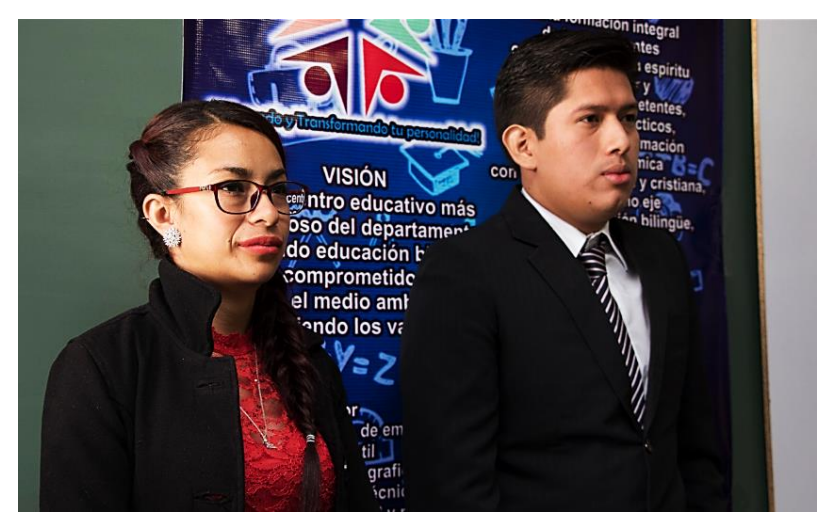

Figura 6 Presentación de proyectos educativos Fuente:(Social, 2018)

\section{Resultados y discusión}

En las siguientes gráficas, se enlistan las competencias profesionales instrumentales, interpersonales y sistémicas, que son desarrolladas y potenciadas mediante el desarrollo de proyectos académicos, basándose en una escala de 0 a $10 \%$, de acuerdo a los objetivos de las diferentes asignaturas y a la evaluación del cumplimiento de los mismos, así como a entrevistas con docentes y estudiantes de las diferentes IES.

En el Gráfico 1 se muestran los porcentajes de desarrollo de las competencias instrumentales, de las que puede observarse que la competencia que mayormente desarrollaron los estudiantes, fue la tolerancia al estrés, debido a que como comentaron, lograron realizar un proyecto de principio a fin desde la etapa de planeación.

En segundo lugar, se visualiza que el compromiso es la competencia interpersonal que desarrollan, debido a que como son parte importante de un equipo de trabajo y toman una responsiva; les queda claro que, al fallar, podrían impactar el resultado final de manera negativa y no se lograrían los objetivos.

Tanto la organización del tiempo como el análisis de problemas, se logran al organizar sus actividades y tiempos; y al analizar con detenimiento las áreas de oportunidad del proyecto planteado y durante su ejecución. $\mathrm{La}$ toma de decisiones es otra de las competencias altamente desarrolladas, en un $87 \%$.
Cabe destacar que la competencia menormente potenciada es la comunicación en un segundo idioma, por lo que se deben reorientar los proyectos estableciendo parámetros de evaluación que consideren la defensa del proyecto de manera oral y escrita para en un segundo idioma, específicamente se plantea que sea en inglés, puesto que es una debilidad de muchas IES, principalmente públicas.

\section{Desarrollo de competencias interpersonales}

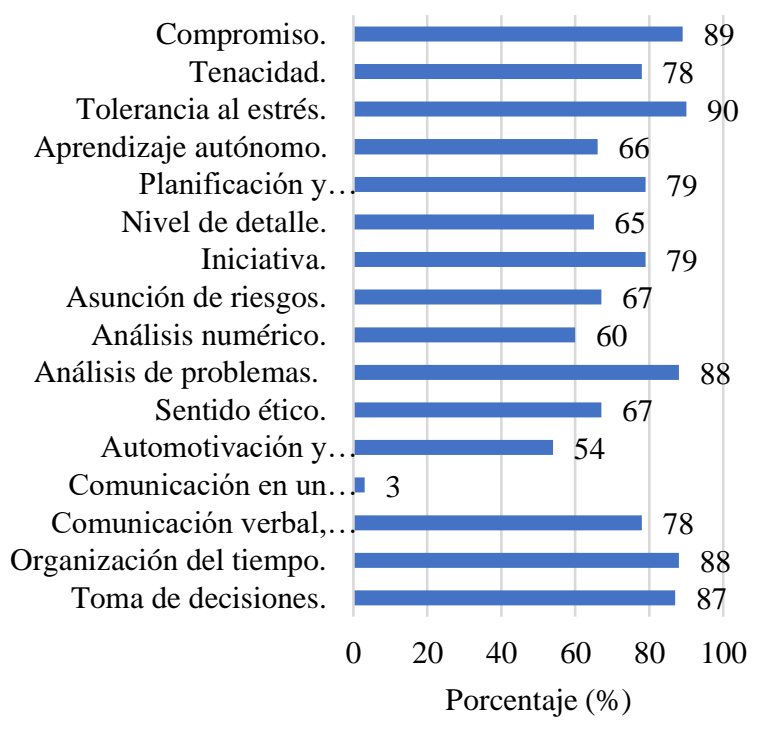

Gráfico 1 Porcentaje de desarrollo de competencias interpersonales

Fuente: elaboración propia

En el Gráfico 2, se muestran las competencias instrumentales, en las cuales sobresale el que los estudiantes al ejecutar proyectos académicos, pueden aplicar los conocimientos a la práctica, lo cual es de suma importancia porque se permite evaluar el nivel de conocimiento que tienen y qué logran hacer con éste. 


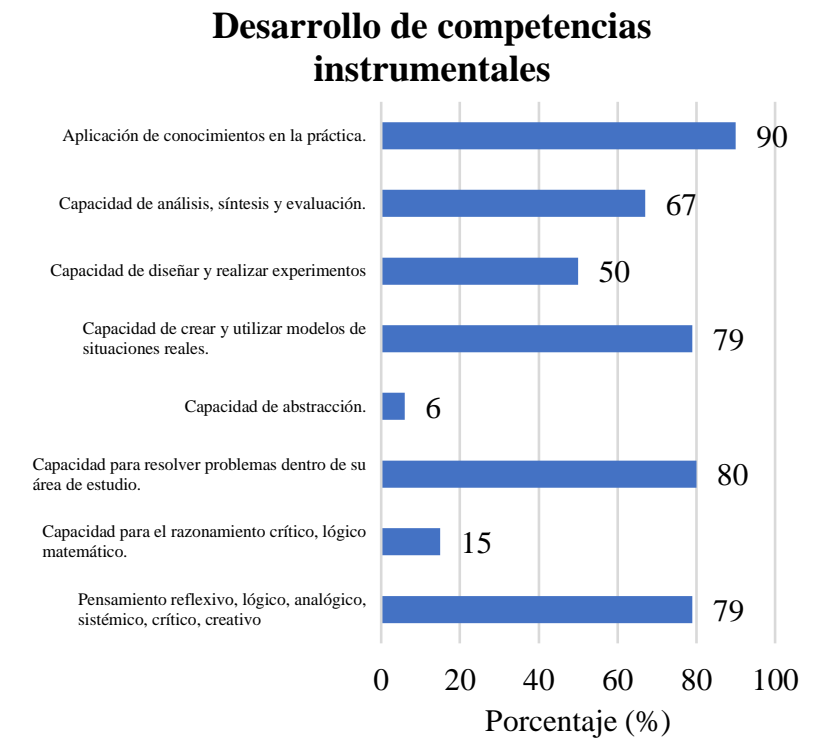

Gráfico 2 Porcentaje de desarrollo de competencias instrumentales

Fuente: elaboración propia

En el Gráfico 3, se observa que la principal competencia sistémica que los estudiantes lograron trabajar es la capacidad de escuchar, argumentando que, partiendo de una buena comunicación, lograron organizarse y ponerse de acuerdo en los diferentes compromisos establecidos como integrantes de un equipo.

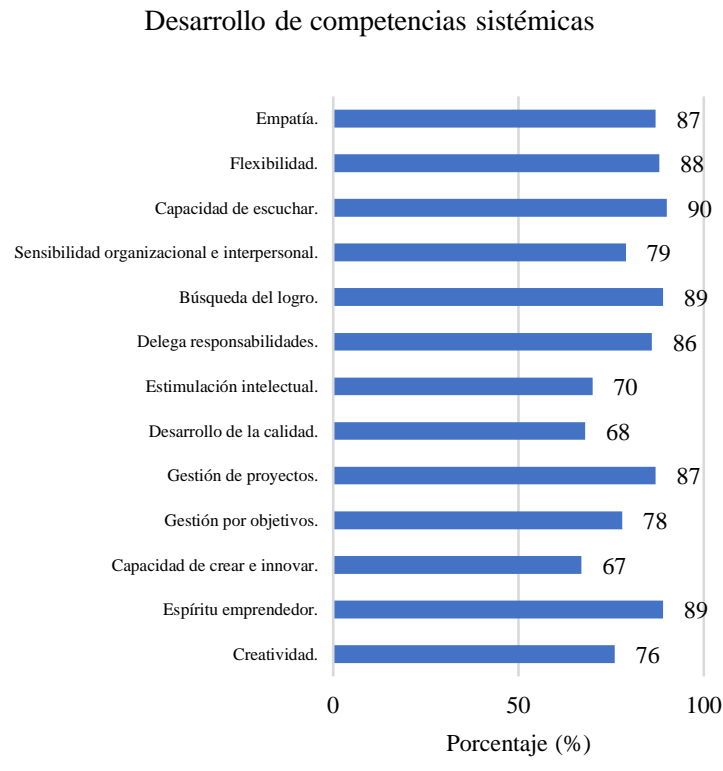

Gráfica 3 Porcentaje de desarrollo de competencias sistémicas

Fuente: elaboración propia

\section{Conclusiones}

La ejecución de proyectos académicos a lo largo de los diferentes momentos formativos de los estudiantes, tienen un impacto en el desarrollo y aplicación de competencias por lo que estudiantes, docentes y autoridades educativas deben promoverlos y presentarlos en diversos escenarios internos y externos mediante la vinculación con otros estudiantes de IES, investigadores, empresas privadas $\mathrm{y}$ organizaciones gubernamentales.

En los ejemplos mostrados, se observa que cada Institución los organiza y establece de manera libre, no existe una estructura definida, pues los proyectos académicos pueden ser simulaciones, prototipos, maquetas, etc. pero todas buscan atender una necesidad e impactar en los distintos sectores estratégicos del país, derivadas de alguna necesidad o problemática, dentro o fuera de la IES, promoviendo que los estudiantes analicen y propongan alternativas de solución evaluando su factibilidad y tomando de decisiones que les permitan obtener resultados cuantificables, guiados por docentes y asesores.

Gracias a la realización de los proyectos académicos, se logró que los estudiantes desarrollaran o potenciaran competencias profesionales permitiendo evaluar los niveles de evolución al aplicar los conocimientos a la práctica y el crecimiento interpersonal, por lo que es una estrategia de enseñanza-aprendizaje que le permitirá aprender de manera autónoma disponiendo de diversas los conocimientos, habilidades y actitudes.

Las tres competencias generales que se desarrollan en mayor medida con los proyectos académicos, son la capacidad de escuchar, la tolerancia al estrés y la capacidad de aplicar los conocimientos a la práctica.

Puesto que solo el $3 \%$ de los proyectos académicos, concretan el desarrollo de competencias de comunicación en un segundo idioma, deben evaluarse los planteamientos y criterios de evaluación para que los estudiantes puedan exponer sus proyectos en idioma inglés. 


\section{Referencias}

$6^{\circ}$ Encuentro de Jóvenes Investigadores en el estado de San Luis Potosí. (2018). Recuperado 12 de septiembre de 2019, de https://crmnoticias.com.mx/concluye-de-formaexitosa-el-6o-encuentro-de-jovenesinvestigadores-en-el-estado-de-san-luis-potosi/

Clares, P. M., \& Samanes, B. E. (2009). Formación basada en competencias. 27, 25.

Estudiantes de Ingeniería presentaron proyectos de negocios-El Amigo de la Marro. (2019). Recuperado 12 de septiembre de 2019, de https://noticias.ufm.edu/estudiantes-ingenieriapresentaron-proyectos-negocios/

Galván, J. J. M., Pérez, P. R. Á., Torres, J. M. D., \& Betancort, H. J. (2019). La orientación académica y profesional del alumnado de la Facultad de Educación: Un elemento transversal del proceso formativo. De los procesos de cambio al cambio con sentido, 2019, ISBN 97884-16471-20-1, págs. 83-92, 83-92. Recuperado de

https://dialnet.unirioja.es/servlet/articulo?codig $\mathrm{o}=6954755$

Jarrín, F., \& María, C. (2013). Plan de negocios Proyecto IESS El Ejido-Manzana 5. Recuperado de http://repositorio.usfq.edu.ec/handle/23000/306 6

Modelo educativo para el siglo XXI: Formación $y$ desarrollo de competencias profesionales. (2018). Recuperado de https://www.tecnm.mx/modeloeducativo

Navarro Soria, I., González Gómez, C., López Monsalve, B., \& Contreras Fontanillo, A. (2019). Aprenentatge cooperatiu basat en projectes i entorns virtuals per a la formació de futurs mestres. Educar, 55(2), 519. https://doi.org/10.5565/rev/educar.935

Ortuño, E. D. L. Á. I. (2019). Diseño educativo de una actividad profesional emergente desde las competencias cognitivas: Mediación de conflictos sociales. Revista Educación, 278-292. https://doi.org/10.15517/revedu.v43i2.33063
Social, C. (2018). Presentación de Proyectos Educativos Institucionales de la Universidad Mesoamericana. Recuperado 12 de septiembre de 2019, de https://www.mesoamericana.edu.gt/presentacio n-de-proyectos-educativos-institucionales/

Tec Superior S.L.P. (2019). Recuperado 7 de septiembre de 2019, de http://www.tecsuperiorslp.edu.mx/

Universidad Tecnológica de la Costa Grande de Guerrero. (2017). Recuperado 11 de septiembre de 2019, de http://www.utcgg.edu.mx/ofertaeducativa/ 